\title{
Design, construction, and implementation of a ground-based solar spectrograph for the national student solar spectrograph competition
}

E. Keeler, D. Moen, C. Peck, C. Zimny, K. Repasky

E. Keeler, D. Moen, C. Peck, C. Zimny, K. Repasky, "Design, construction, and implementation of a ground-based solar spectrograph for the national student solar spectrograph competition," Proc. SPIE 8481, Optics Education and Outreach II, 84810Y (18 October 2012); doi: 10.1117/12.965320

Event: SPIE Optical Engineering + Applications, 2012, San Diego, California, United States 


\title{
DESIGN, CONSTRUCTION, and IMPLEMENTATION of a GROUND- BASED SOLAR SPECTROGRAPH for the NATIONAL STUDENT SOLAR SPECTROGRAPH COMPETITION
}

\author{
E. Keeler, D. Moen, C. Peck, C. Zimny, K. Repasky \\ Montana State University, Bozeman, Montana 59717
}

\begin{abstract}
A solar spectrograph is an instrument that takes incoming sunlight over a majority of the electromagnetic spectrum and separates the light into its constituent frequency components, or spectrum. The components are then sent to a detector that measures intensity, which reveals the location of spectral properties of the light such as absorption and emission lines. The National Student Solar Spectrograph Competition (NSSSC) is a Montana Space Grant Consortium sponsored competition where undergraduate student teams from across the country design, build, and implement a ground-based solar spectrograph to perform any solar related task and demonstrate their spectrographs for the competition in May 2012 in Bozeman, MT. Each team is given a 2,000-dollar budget to build their spectrograph, which cannot be exceeded, and all spectrographs must follow regulations in the NSSSC guidelines. This team designed the spectrograph to be capable of imaging the sun across the visible spectrum using spatial filters and a standard photo detector rather than a traditional charge-coupled device due to budget limitations. The spectrograph analyzes the spectrum of small sections of the sun to determine how the spectrum varies across solar features such as the corona, active regions, and quiet regions. In addition to solar imaging, the spectrograph will also analyze atmospheric absorption of the solar spectrum by comparing the measured spectrum to the theoretical spectrum calculated from the blackbody equation.
\end{abstract}

Keywords: Spectrograph Design, Solar Imaging, National Student Solar Spectrograph Competition (NSSSC)

\section{INTRODUCTION}

A solar spectrograph is an instrument that takes incoming sunlight over a majority of the electromagnetic spectrum and separates the light into its constituent frequency components, or spectrum. The components are then sent to a detector that measures intensity, which reveals the location of spectral properties of the light such as absorption and emission lines. The National Student Solar Spectrograph Competition (NSSSC) is a Montana Space Grant Consortium sponsored competition where undergraduate student teams from across the country design, build, and implement a ground-based solar spectrograph. The competition begins in September 2011 and ends with the final competition in May 2012 where students demonstrate their spectrographs in Bozeman, MT. Each team is given a $\$ 2,000$ budget to build their spectrograph, which cannot be exceeded and all spectrographs must follow regulations in the NSSSC guidelines. Additionally, each team may have three to six undergraduate students and must have one faculty advisor.

Each team in the NSSSC has four primary goals:

- Determine an experimental use for the spectrograph.

- Design and build the spectrograph to best carry out the experiment.

- Calibrate spectrograph to make pre-defined observations of the solar spectrum and demonstrate resolution and sensitivity of the device.

- Observe and analyze experimental results and repeat observations and results at the competition.

Students can develop any experimental purpose for their spectrograph provided it follows the guidelines of the competition. Therefore, students learn the significance of independent design and implementation of an experiment throughout the academic year while developing teamwork skills. Each student in the team will focus on different parts of the project, but all members will be involved in every phase.

Optics Education and Outreach II, edited by G. Groot Gregory, Proc. of SPIE Vol. 8481

84810Y · ( 2012 SPIE · CCC code: 0277-786/12/\$18 · doi: 10.1117/12.965320 


\section{BACKGROUND}

The group consists of an interdisciplinary team, which drives each member to work together and bring specific skills to the table. All required knowledge for the competition should be rooted well in the education each member has received in their past three years of training in their fields. Two electrical engineers (Ethan Keeler and Drew Moen) will join the team to handle most of the electronics and data collection while the physicist (Courtney Peck) will focus on the understanding of the solar spectrum and optical components. Additionally, the team added a mechanical engineering student (Chris Zimny) to create adequate housing for the spectrograph along with the construction of custom mounts and motor systems. However, all team members will collaborate on all parts of the project including instrument understanding, calculations related to the optical components, and assembling and programming the spectrograph, which increases each member's skills in other areas.

Throughout the 2011 summer, the team met once a week to begin learning about the components of a spectrograph, the many options in design, and how to mathematically model its components. To be more specific about the necessary general understanding of spectrograph principles, the team focused on determining the basic design of a spectrograph, calculating reflection angles off of a diffraction grating, determining size and focal length requirements of the lenses to obtain particular results, and calculating theoretical spectra of the Sun above earth's atmosphere. Although the physicist was involved with all aspects during the 2011 summer, her main focus was calculation of the theoretical solar spectrum at the top of earth's atmosphere by treating the sun as a blackbody radiator. From this, she decided on the experimental use for the spectrograph. The proposed experiment was to measure the solar spectra as a function of time and determining the atmospheric absorption by comparing measurements to the theoretical solar spectrum calculations. The difference between the two spectra is the atmospheric absorption. The second, more complex, goal of the project was to create an imaging spectrograph without the use of a CCD, rather the spectrograph would use spatial filtering to obtain the same functionality.

\section{METHODOLOGY}

\subsection{Experimental use}

The main starting point of the project was to determine an experimental use for the spectrograph. This involves knowing the limitations in resolution and sensitivity of the device within the allotted budget as well as an understanding of the solar spectrum and earth's atmosphere.

One determined experimental use was to analyze the effect of atmospheric absorption of the solar spectrum as seen at a particular location. The group also considered a second experimental use for the spectrograph that would be used simultaneously with the previously described experiment. This experiment would measure solar compositions by observing thermal splitting of the spectral lines. To determine if this experimental use was appropriate for the team, Courtney Peck calculated the amount of thermal splitting and determined that it would not be possible to make a spectrograph within the budget with the necessary resolution and sensitivity. The team decided to perform solar imaging with the spectrograph to observe spectral differences across solar features instead. The team also extended this goal to include analyzing atmospheric absorption by viewing the different spectral features.

\subsection{Design and construction}

Once experimental uses were determined, the group moved on to design and construction of the device. Because an optical detector only works over a particular wavelength, all experimental uses had to be within the same wavelength range of the single detector. Design of the spectrograph began with extensive theoretical calculations of the components. 
One key role was to work through theoretical calculations of components to determine a spectrum that will be observed at the detector. This was accomplished by correcting a theoretical spectrum using the resolution of lenses and the response function of the detector to adjust the spectrum for these components. The role also determined second order corrections that occurred at angles of the diffraction grating and determined if these corrections were significant in our detection or not.

As soon as theoretical design had been perfected, the team moved onto purchasing equipment to meet the budget constraints. A light-tight and shock resistant box needed to be constructed to house all the optical equipment. Any light leaked into the box would distort the solar spectra causing noisy results and any noticeable shock onto the box would misalign the optical equipment. For this stage of the project, the mechanical engineer learned how to weld the light-tight and shock resistant box.

After all the components had been received and the box built, all of the equipment could then be assembled. At this stage, the detector and motor to tilt the diffraction grating was programmed by a computer to collect data. When this was completed, the spectrograph was in working condition and ready for calibration.

\subsection{Calibration}

Calibration involves testing the equipment to make sure it matches specifications, correcting the detected solar spectra for equipment responsivity and resolution, and making additional corrections for the measured spectra to match the known spectra. Additionally, it is at this stage where the device's resolution and sensitivity could be determined and compared to theoretical values. In this stage, the theoretical values could be calculated and compared to the actual values measured. If the values do not correlate as well as one would like, the team needs to go back and adjust parameters for the detector to more closely relate these values.

\subsection{Observation and analysis}

Once calibration was complete, the spectrograph could then perform the proposed experiments. This stage involved collecting data over periods of time similar to the allotted time for the competition, approximately four hours. Once the data was collected, it could be analyzed. Analysis would depend on the types of experiments the team decided to do and what variables the team wanted to focus on.

\section{DESIGN}

Given the guidelines and experimental use of the spectrograph, the team constrained the design to each of the scientific objectives as outlined below.

For atmospheric absorption:

- The spectrograph must be capable of a minimum resolution of $10 \mathrm{~nm}$ to resolve major absorption features. For solar imaging

- The solar image at the image plane must be divided into at least 100 pixels to achieve the desired image resolution.

- The solar image at the image plane must be large enough to align with the optics and pinhole and for the pinhole to be relatively inexpensive.

The following subsections describe the optical design that was chosen to best implement these experiments and their requirements.

\subsection{Entrance optics}

The designed system gathers light using a $50.8 \mathrm{~mm}$ lens. The lens uses a large focal length of $1 \mathrm{~m}$ to make the sun's image at the image plane (focus of lens) relatively large. This is advantageous for this project because the linear motors used for spatial filtering (for imaging applications) can have a large step size and the pinhole can be fairly large, making them more inexpensive. The size of the sun that will be imaged was determined using Equation 4.1. 


$$
h=\Theta f .
$$

Theta is the angular size of the sun in the sky, and $f$ is the focal length of the first lens. In order to achieve a size of the sun that would be easily imaged, the largest focal length commonly available was chosen where $f=1000 \mathrm{~mm}=1$ $\mathrm{m}$. The angular size of the sun is 0.53 degrees, yielding a height of the sun at the image plane of $9.3 \mathrm{~mm}$. This solar size will allow the use of a pinhole that is large and will make the experiment easier to align.

The intake lens will sit at a 45 degree angle to the ground (competition design requirement) and will send the light to a mirror close to the ground $0.75 \mathrm{~m}$ away from the lens. The mirror will reflect the light in the plane of the spectrograph, 45 degrees below the incident beam to the slits $0.25 \mathrm{~m}$ away to propagate horizontally. The pinhole will be placed at the image plane of the lens that focuses the incoming sunlight. The lens chosen has a focal length of $1 \mathrm{~m}$ as described above and a $50.8 \mathrm{~mm}$ diameter to allow for large intensities into the spectrograph.

\subsection{Pinhole and slit calculations}

As stated previously, the focal length of the first lens was chosen to have a large focal length to create a solar image at the image plane that can be broken into roughly 100 pixels on the solar image using a large pinhole to save money. The focal length of the first lens is $1000 \mathrm{~mm}$, creating an image at the image plane of approximately $9.3 \mathrm{~mm}$. The width of the slit for measuring atmospheric absorption and diameter of the pinhole for solar imaging are needed to be around $0.6 \mathrm{~mm}$ to $1 \mathrm{~mm}$ to create the desired resolution and number of pixels on the sun for each experiment. The exact value of the pinhole size was chosen to align with the step size of the linear motors used to move the pinhole across the solar image, and the width of the slit was chosen to be the same as the diameter of the pinhole to keep the optics consistent without making changes to the optics to change an experiment. The step size of the motor is 0.0315 " = $0.8 \mathrm{~mm}$. The slit width and pinhole diameter were therefore chosen to be $0.8 \mathrm{~mm}$ to coincide with the step size, which also fell in the range of necessary size. The rest of the lenses necessary were calculated under these conditions.

Using the numerical aperture formulas, the size and focal length of the second lens can be found to re-collimate the light for the grating. The numerical aperture was found using Equation 4.2.

$$
N_{1}=\frac{f_{1}}{D_{1}}=N_{2}=\frac{f_{2}}{D_{2}} .
$$

$f_{1}$ is the focal length of the first lens, $D_{1}$ is the diameter of the first lens, and $f_{2}$ is the focal length of the second lens. Because of the changing direction of the rays depicted in the previous figures, $D_{2}$ is the beam width of the light from each pixel, rather than the diameter of the second lens.

The central pixel on the sun should be aligned centrally to all the optics. However, the pixels above or below that pixel are off axis and introduce some necessary optical considerations. Using trigonometry between the first and second lens, and the off-axis pixel height of $9.35 \mathrm{~mm} / 2=4.68 \mathrm{~mm}$, the diameter of the second lens would need to be 1 " or $25.4 \mathrm{~mm}$ in order to capture the light from all pixels. Using the 1" lens allows for a maximum off-axis pixel height of $5.08 \mathrm{~mm}$, leaving room for error in misalignment of the optics without losing light from a pixel. This requires from the relationship in Equation 4.2 that $f_{2}=250 \mathrm{~mm}$ in order for the beams from each pixel to be collimated after the second lens.

\subsection{Objective lens}

The collimated beams from the pixels when they reach the diffraction grating will have diverged from the center pixel as a function of the output angle from the second lens. The most extreme angle of divergence will result from the most off-axis pixel at $5.08 \mathrm{~mm}$ and has a value of around 1 degree. The amount of divergence through the distance from the second lens to the third lens determines the diameter of the third lens so that no light from the pixels is lost. To allow for separation of the wavelengths off of the diffraction grating, the distance from the diffraction grating to the third lens was chosen to be large. This enabled the use of a large (adjustable), and therefore inexpensive, slit placed before the detector to control the resolution of the spectrograph. The distance chosen was $1000 \mathrm{~mm}$. The total divergence of the most extreme pixel over this length will still be collected by the third lens if the diameter of the lens is 2". The focal length of this lens was chosen so that the light from all pixels would be focused onto the active region of the 
detector (3.6 mm X $3.6 \mathrm{~mm}$ ), preferably, without moving the detector with a motor. The focal length that achieved this needed to be under $80 \mathrm{~mm}$. Therefore, the third lens focal length was chosen to be a more standard length of $75 \mathrm{~mm}$. The slit choice considerations are evaluated under the section on resolution.

\subsection{Optical summary}

The following diagrams show the rays for the center pixel and the most extreme pixel, to show that all the light from all pixels on the sun will be focused onto the detector.

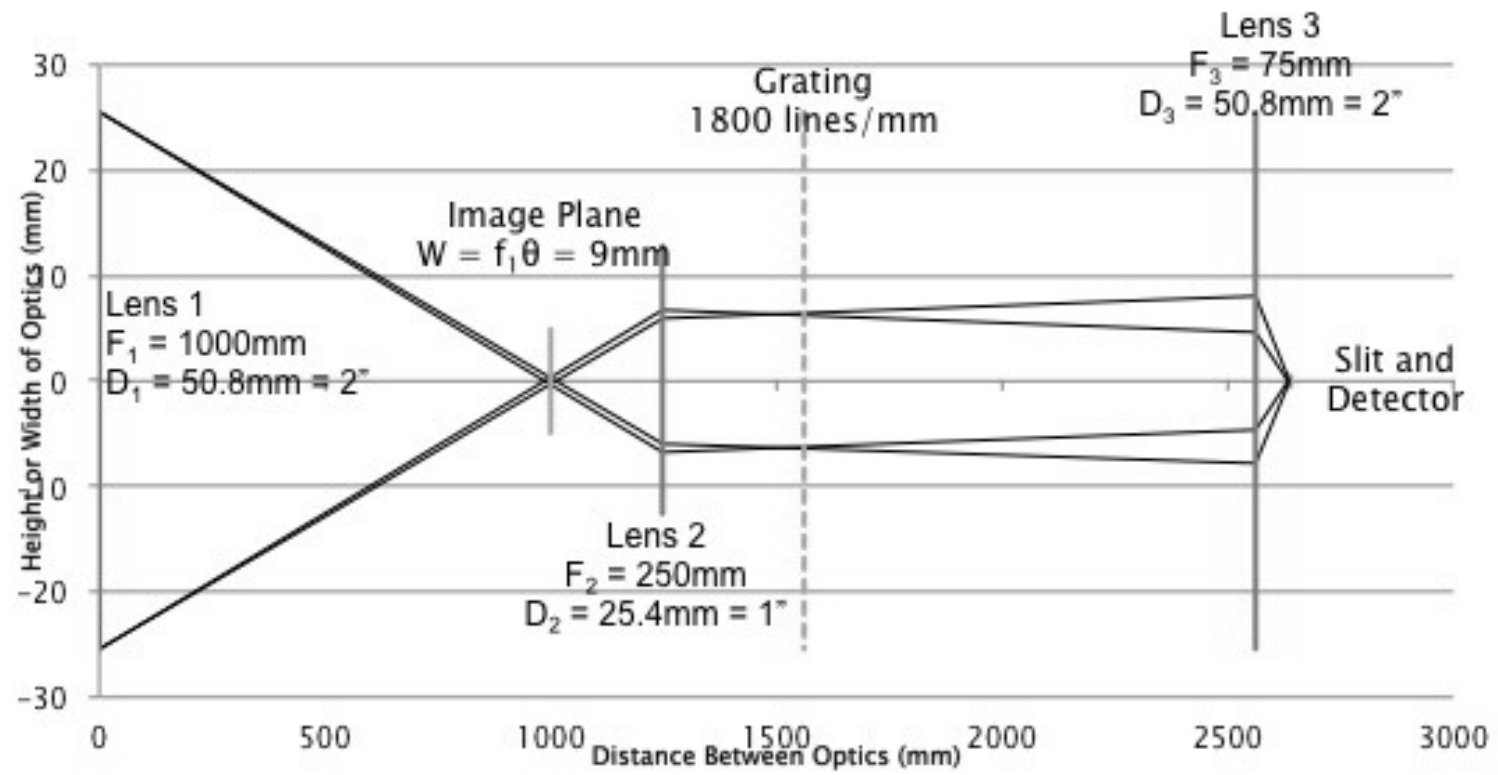

Figure 4.1: Ray diagram showing the most extreme rays for the pinhole centered on the optical axis. Note how all light rays are passed through all the optics and focused onto the detector.

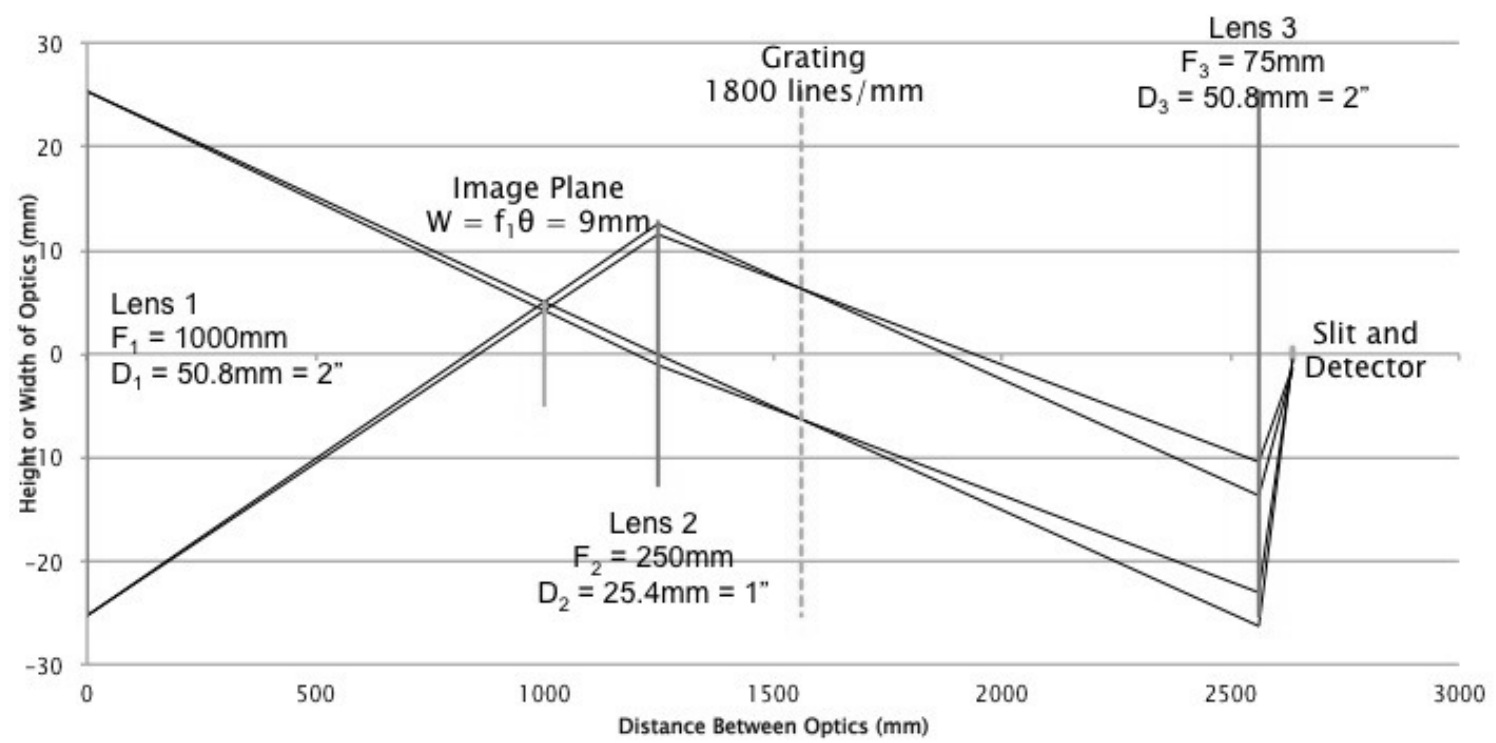

Figure 4.2: Ray diagram showing the most extreme rays for the pinhole off-centered at $5.08 \mathrm{~mm}$ on the optical axis. Note how all light rays are passed through all the optics and focused onto the detector. 


\subsection{Detector}

The photodiode for the project system has an active area of $3.6 \mathrm{~mm}$ by $3.6 \mathrm{~mm}$, and it comes with a calibrated responsivity plot for better accuracy. The same photodiode can be found for cheaper, but it would not come with the calibrated responsivity plot. This specialized responsivity plot was deemed important since it would be too difficult to calibrate such data from any available lab equipment. It can measure a desired wavelength range of 350 to $1100 \mathrm{~nm}$. The photodiode could potentially be a limiting factor in the amount of light intensity being detected due to its dark current of $20 \mathrm{nA}$. If the signal intensity is too low, the dark current will act as a noise floor, blocking the signal. The maximum forward and reverse currents are $5 \mathrm{~mA}$. This means if the signal intensity is too high, the photodiode will saturate into a nonlinear region. Using this information and the responsivity data, it can be shown that the minimum allowable intensity on the detector is $0.4 \mu \mathrm{W}$ and the maximum allowable intensity on the detector is $8.33 \mathrm{~mW}$.

A possible alternative to the photodiode would be some sort of photo-detector or CCD. These ideas were quickly abandoned because of the price constraint. Since the calibrated photodiode suited the requirements of the project design, and only cost about $\$ 145$, it was a good choice for the project. A trans-impedance amplifier is also attached to the photodiode to allow a microprocessor to sample a signal voltage.

\subsection{System electronics}

As with most modern systems, a microcontroller will oversee the control and operation of the spectrograph system. However, the microcontroller will operate subordinate to the directives of a computer. The computer will gather the collected data and will interface with users to operate the spectrograph. The following subsections describe the electronics of the system.

\subsection{Analog-to-digital converter}

In order to ensure that the system was not limited by analog-to-digital resolution, the project utilized a ten bit (10) A/D converter to digitize the signal from the photodiode. This converter should give adequate resolution to see absorption features.

In order to convert the current signal of the photodiode to a voltage for digitization by the $A / D$, the project proposes the following trans-impedance amplifier circuit. Since the maximum current from the photodiode could be as high as approximately $57.5 \mu \mathrm{A}$ and to utilize the $\mathrm{A} / \mathrm{D}$ range to the fullest, the circuit implements a gain of 80,000 yielding a maximum voltage to the converter of $4.6 \mathrm{~V}\left(V_{o}\right)$. Figure 6.8.2.1 shows the circuit layout. 


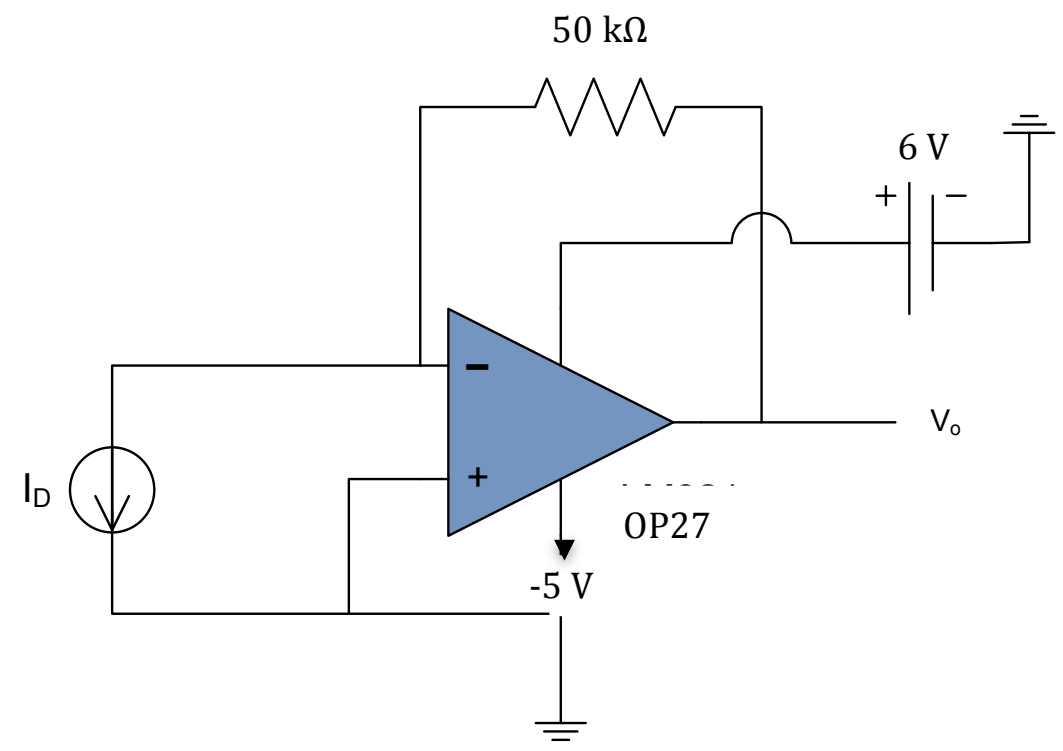

Figure 4.3: Trans-Impedance Amplifier Circuit. This circuit takes the current produced by the photodiode and transfers the signal to voltage which can be sampled by an A/D converter.

To be on the safe side without missing information outside of 0 to $4.6 \mathrm{~V}$ range, the project biased the operational amplifier slightly higher than 5 volts and lower than 0 volts to ensure that the circuit output can realize the full 0 to 5 volt range (without worrying about rail limitations). This translates to a voltage step size of $48.8 \mu \mathrm{V}$ and a current resolution of $61.0 \mathrm{nA}$. The project cannot go much less than this current resolution since the 'dark current' (the current always present with or without light) is on the order of $20 \mathrm{nA}$.

\subsection{Casing and layout}

A 6.5 " tall box will be constructed from 14 gage sheet steel to house the spectrograph optics and electronics. This gauge of steel was chosen for its stiffness characteristics: it will resist bending from the interior components and vibration from any roof mounted HVAC system that might be operating during the competition. The input lens is mounted at the top of a 23 " long section of 2" PVC conduit mounted at a $45^{\circ}$ degree angle to the ground such that it lines up with the light reflected by the heliostat (a device that tracks the sun and keeps light focused at a specific spot on the ground). This pipe input design was chosen to restrict the ambient light entering the system. PVC conduit was chosen as a material for its low density, low cost, and availability. Within this entrance tube, a 3" long lens mount will hold the 2" diameter entrance optic; the length of this mount is to allow for minor adjustments while aligning the optics system. After the light passes through the entrance tube and into the spectrograph interior, a mirror will then reflect the light into the optics plane of the remaining components. The height of this plane is 4" above the base of the spectrograph and parallels the ground. To prevent stray light from affecting result accuracy, the interior of the box is completely black so that superfluous wavelengths may be absorbed. To allow easy access to the box interior, a panel on the top surface of the box is removable. Padding around the edges of this panel keeps the box light tight. Two handles are also attached to the side in order to ease the process of spectrograph travel.

The electronics compartments are located in the gaps between the path of the light. These sections are blocked off from the rest of the interior to allow heat to diffuse to the outside of the box and to prevent the light emitted by the electronics from interfering with the measurements. One such compartment nearer the grating will contain the power supplies, drivers, and controllers (Figure 4.4). The second compartment, toward the detector, will house the microprocessor. The reason for separate compartments is that the data collection equipment needs to be as close to the detector as possible to reduce line noise. The compartments are cooled via natural convection; holes are drilled to the 
exterior of the box to facilitate heat dissipation. In addition, three ports were machined to accommodate a USB, electrical input, and power switch. All component mounting is on the bottom surface, paralleling the ground.

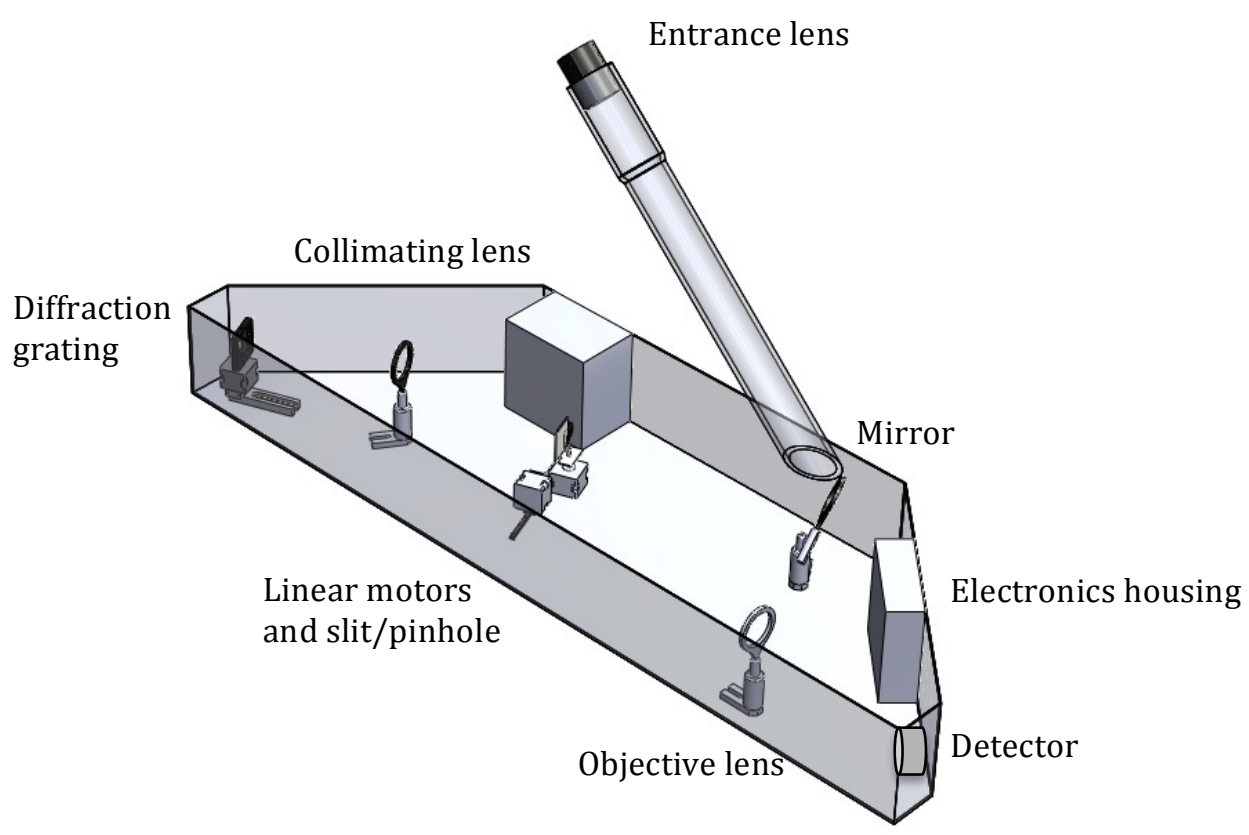

Figure 4.4: System Physical Layout, this depiction shows the box and its interior (with the lid removed).

To effectively work for the competition, the spectrograph must take light coming in at a $45^{\circ}$ angle; in order to make this design more versatile, though, the current design allows for this angle to be manipulated by adjusting the base supports of the spectrograph. The spectrograph is supported by four adjustable legs. Each leg on the box is a $3 / 8^{\prime}$, diameter threaded rod with a lock nut for a foot. These feet are able to move in and out of the box providing $\pm 5^{\circ}$ of motion to the entire box and accommodate the use of the device on uneven surfaces. To prevent slip between the foot and the ground, a rubber pad was fastened to the bottom of the feet. The final weight is near 50 pounds.

\subsection{Motor System}

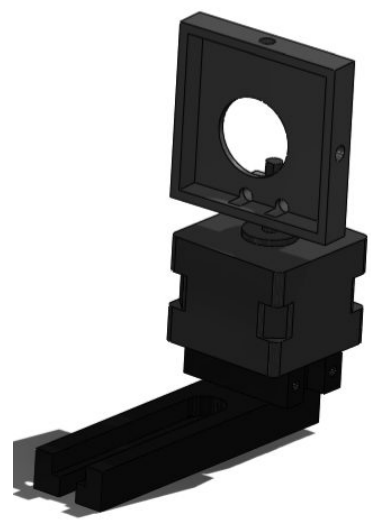

Figure 4.5: Rotational Motor Assembly, this assembly mounted the grating and allowed a rotational motor (the base in the diagram) to rotate the grating to enable spectral measurements.

The rotational motor is the centerpiece of the rotational motor assembly that will control the grating positioning (Figure 4.5). A two-piece base mount (black, bottom), the rotational motor (gray, middle), a grating mount (gray, top), 
and the grating (not shown) comprise this system. The grating mount portion is constructed from aluminum. This choice was made with regards for aluminum's machinability. The grating is mounted in the top piece by two set screws. The hole in the mount back allows the grating to be removed easily without fear of touching the delicate reflective side. A set screw in the back of the mount enables a solid connection between the mount and the rotational motor shaft. The base mount in black was constructed in two pieces to allow for rotational and translational displacement of the entire system as needed. It should be noted that the rotational motor system does not allow for vertical movement. As such, the plane of light within the spectrograph case was set by this apparatus to be 4 " above the bottom of the case.

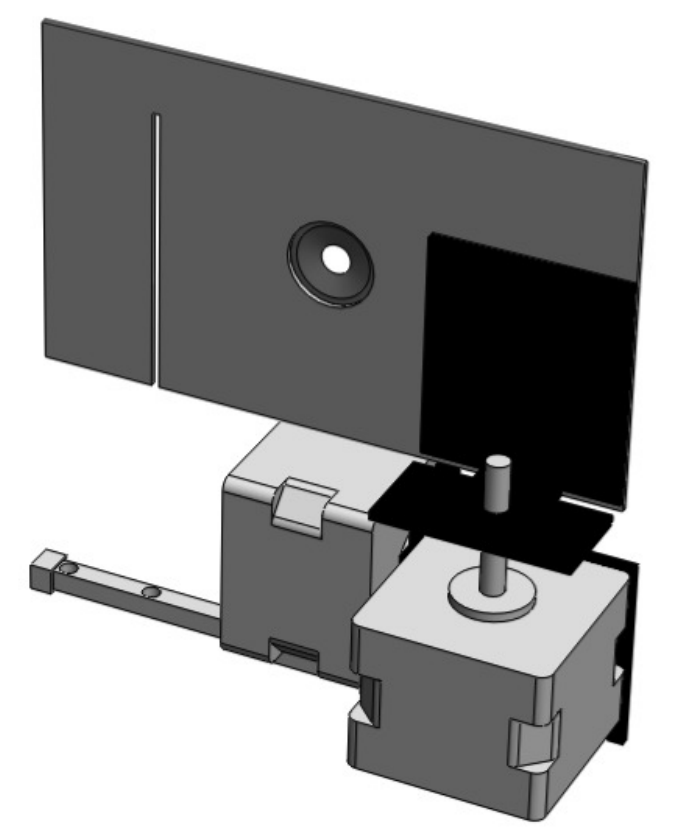

Figure 4.6 Linear Motor System, this assembly resided at the image plane create by the external intake lens. The pinhole could be moved in the X-Y plane to move the pixel across the sun's image. This would provide the necessary spatial filtering and allow for imaging functionality.

These motors are outfitted with guides and sliding platforms manufactured by the design team. The guides are merely M3 threaded rod cut to the appropriate length and inserted into the factory-provided M3 threaded holes. The platforms are constructed by attaching M5 nuts to 14 gauge sheet metal bent at a $90^{\circ}$ angle; a clay epoxy was used for joining due to its malleability in application and strength after curing. The pinhole is mounted in an aluminum bracket manufactured in house, and a set screw is used to hold the pinhole in place. This mount will then be attached to the stage as shown in Figure 4.6. The following components are shown: linear stage (black), linear motor (gray boxes), platforms (light gray), and pinhole mount (medium gray).

Each motor is individually actuated by a separate motor driver. The drivers are situated in the electronics box along with the power supply for each driver and motor. The linear motor system is mounted on a purchased linear motion guide as depicted. This allows the linear motor system to slide out of the sun's path to allow for the alternative science application (the study of absorption using just the slit). This sliding is done manually through an actuator in the side of the box. Any holes made to the exterior casing must be carefully sealed in order to prevent extraneous light from entering the system. 


\subsection{Budget}

\begin{tabular}{|c|c|c|c|}
\hline Description & Part Number/ Company & Price & Quantity \\
\hline Aperture Lens & LB1859/ThorLabs & 30.50 & 1 \\
\hline Collimating Lens & LB1056/ThorLabs & 20.70 & 1 \\
\hline Objective Lens & LB1309/ThorLabs & 31.40 & 1 \\
\hline Mirror & PF20-03-G01/ThorLabs & 95.90 & 1 \\
\hline $800 \mu \mathrm{m}$ Pinhole & Custom/National Aperture Inc. & 59.00 & 1 \\
\hline Adjustable Slit & VA100/ThorLabs & 248.00 & 1 \\
\hline Photodiode & FDS100-CAL/ThorLabs & 146.60 & 1 \\
\hline Photodiode Power Supply & TOL-08269/Sparkfun Electronics & 5.95 & 1 \\
\hline Grating & 53-*-290R/Richardson Grating & Free & 1 \\
\hline Translational Stage & RSR 7ZM/THK Linear Motion & 50.20 & 1 \\
\hline Microcontroller & DEV-11061/Sparkfun Electronics & 58.95 & 1 \\
\hline USB to RS-485 Converter & BOB-09822/SparkFun Electronics & 19.95 & 1 \\
\hline Linear Motor & $\begin{array}{l}\text { 17AY102S-LW4-C/Anaheim } \\
\text { Automation }\end{array}$ & 30.00 & 2 \\
\hline Linear Stepper Motor Driver & ROB-10267/Sparkfun Electronics & 14.95 & 4 \\
\hline Linear Motor Power Supply & PS-1228/All Electronics & 10.50 & 2 \\
\hline Rotational Motor/Driver & 17MDSI102S/Anaheim Automation & 177.00 & 1 \\
\hline Rotational Power Supply & PSAM24V2.7A/Anaheim Automation & 100.00 & 1 \\
\hline Sheet Metal & Pacific Steel \& Recycle & 75.51 & 1 \\
\hline Lens Posts/Mounts & ThorLabs & 311.25 & 1 \\
\hline Trivial Case Materials & Local Supplier & 60.00 & 1 \\
\hline 20ft USB Cable & Local Supplier & 20.00 & 1 \\
\hline Trivial Electronics & Local Supplier & 130.00 & 1 \\
\hline Shipping Fees & - & 90.23 & 1 \\
\hline Extra & & 128.06 & 1 \\
\hline Total & & \multicolumn{2}{|l|}{2000} \\
\hline
\end{tabular}

Table 4.1: Budget, this table represents an itemized final budget for the project and demonstrates that the project met the competitions budget constraints.

The spectrograph design was well under budget as shown in Table 4.1, which allowed for necessary padding should components needed redesign or should there have been any unexpected problems.

\subsection{Completed prototype}

From the preceding spectrograph design, the project completed the proposed design into a working prototype. The following section discusses the completion of the spectrograph along with testing plans and implementation of these plans and data analysis. The completed spectrograph design is shown below including the location of all the optics and electronics boxes. 


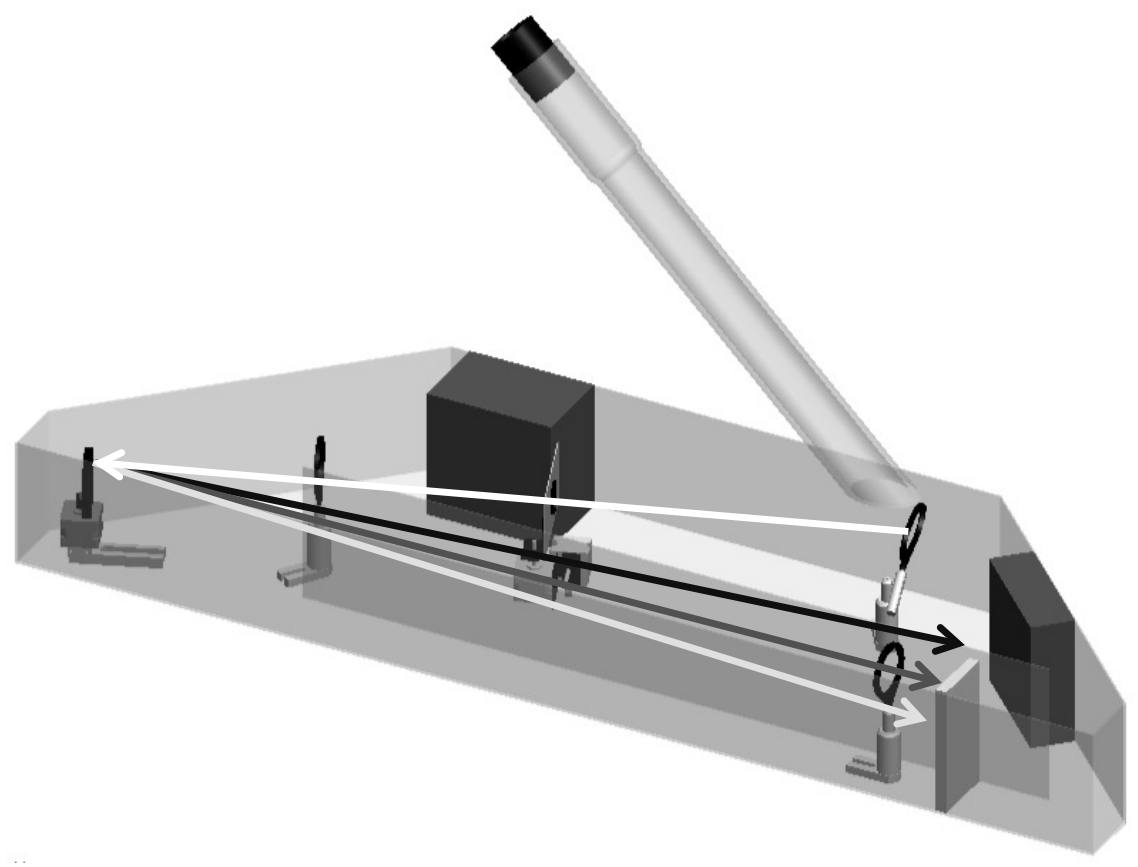

Figure 4.7: Virtual Model of the Completed Spectrograph without Lid, this diagram shows the spectrograph in its entirety; it also traces out the path of the propagating light and follows it to the grating where it spreads into its spectrum.

The picture below shows the spectrograph assembled with all of the optics and electronics. The wires were organized to not obstruct the optical pathway or interfere with any moving parts in the system.

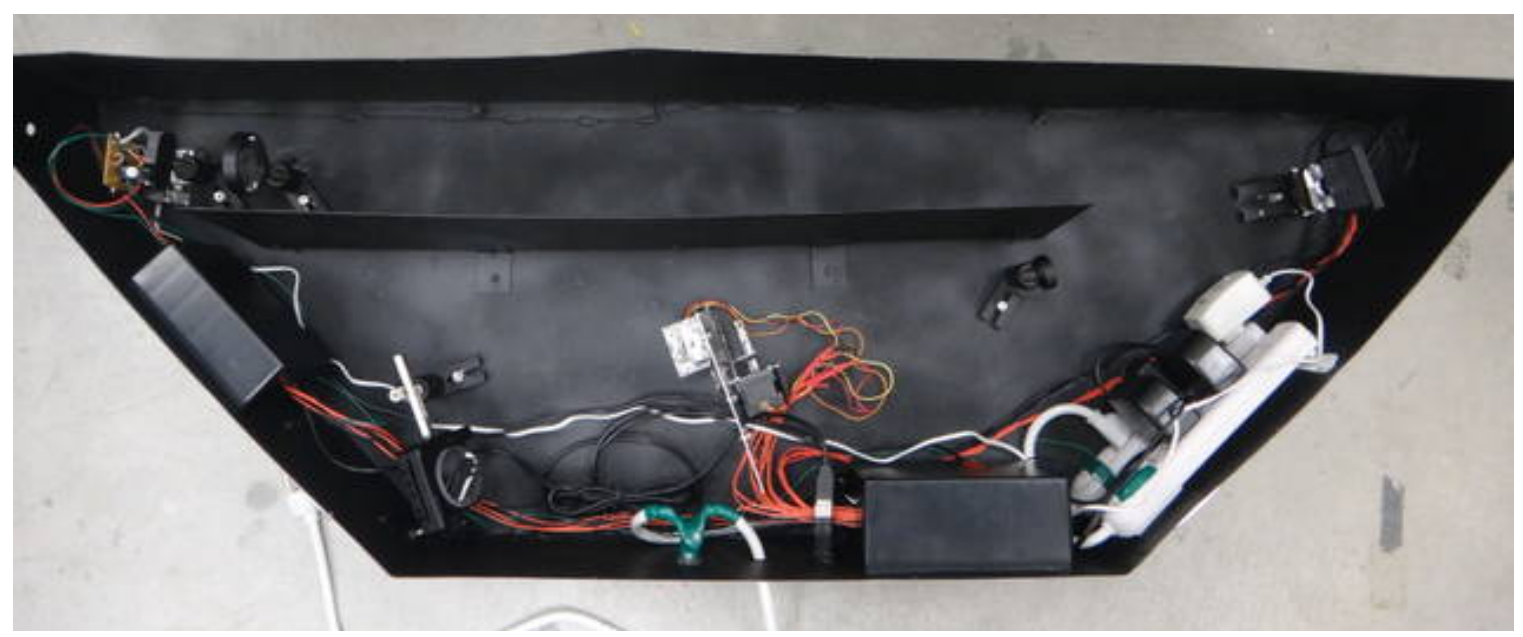

Figure 4.8: Assembled Spectrograph with Optics and Electronics without Lid or Lens Tube, this picture shows the true fabricated box and the many components that it houses.

\section{DATA COLLECTION AND ANALYSIS}

Upon completing assembly of the project, the next phase of the project was testing. The testing plan utilizes several different light sources to confirm spectral measurements and resolution with a spectrum analyzer. 


\subsection{Alignment with laser}

Upon assembly of the optics into the box, the optical components are aligned using a laser light source, usually a $632.8 \mathrm{~nm} \mathrm{He}-\mathrm{Ne}$ laser. To mimic the angle that will be used during the competition, the He-Ne laser was set at a $45^{\circ}$ in the downward direction and the spectrograph was arranged such that the laser light was set in the center of the intake lens of the spectrograph. Then, the mirror was positioned so that when looking into the intake lens, only the mirror is visible, ensuring all the light from the intake lens is passed through the system. Additionally, the spectrograph was adjusted slightly to allow the laser light to pass directly through the lens tube and onto the center of the mirror.

Once the beam was centered on the mirror, the pinhole at the image plane was set to the centered position. The mirror was then tilted using the tilting knobs on the mirror mount to aim the laser beam directly through the pinhole. With the collimating lens moved out of the optical path, the beam is then aligned centered onto the grating, which has a fixed height. Then, the second lens was moved back into the pathway at its appropriate focal length from the image plane and positioned so that the beam passes directly through the center and does not change the position of the beam on the grating

After the beam was aligned onto the grating, the grating was tilted using a shim under the mount to align it onto the detector. The slit and objective lens were aligned so that the centers are at the same heights as the grating center height. To do this, the objective lens was moved out of the optical pathway, and the shim was positioned such that the beam lands directly on the center of the slit, where the detector is positioned directly behind. Then, the objective lens was positioned in the optical path so that the beam is centered on the lens.

\subsection{Testing with tungsten light source}

After finishing the alignment of the optics using the laser light source, the spectrograph was tested using an inlab light source. The light source used in these tests was a tungsten light. To test with this light source, the laser was replaced with the lamp and a lens to collimate the light from the lamp to better mimic the collimated light that comes from the sun. After collimating the light and sending it to the spectrograph, it was then analyzed using either the spectrum analyzer or the electronic system designed for the project. Images from each of these sources spectra, respectively, are shown below.
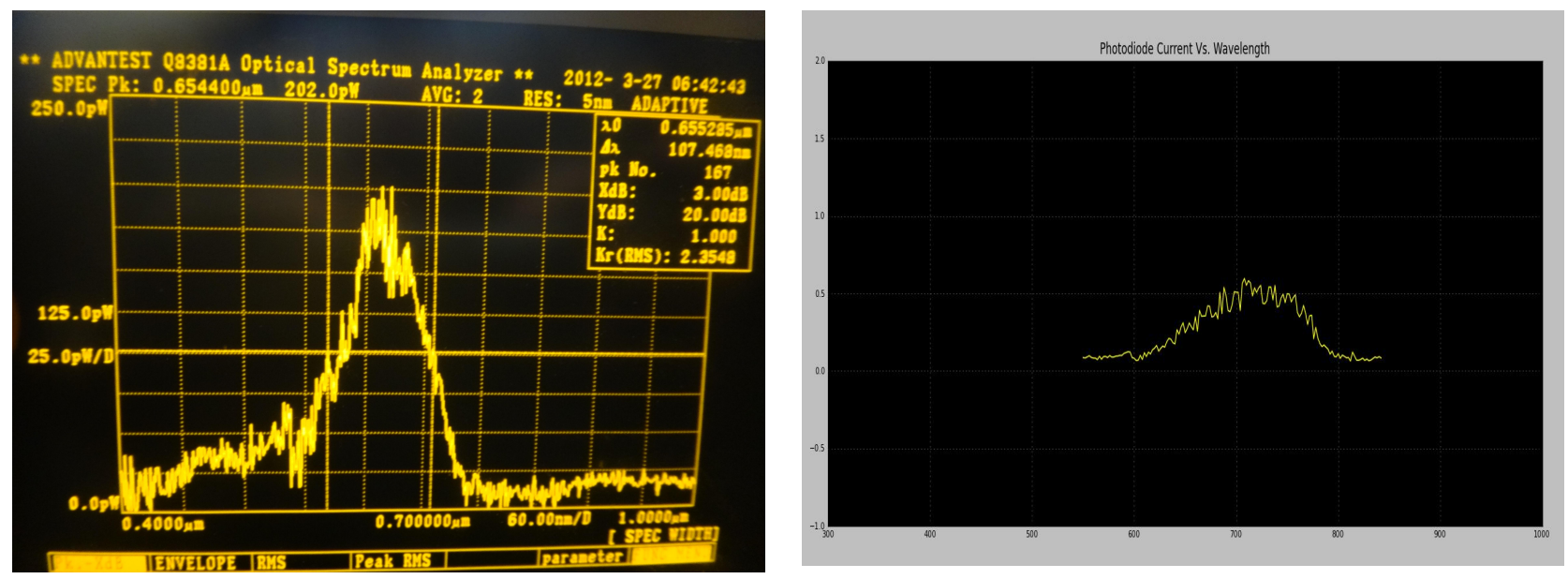

Figure 5.1: Spectra of tungsten light sources. (Left) Spectra as seen on spectrum analyzer. (Right) Spectra as seen with spectrograph data collection system.

\subsection{Testing with laser light}

To determine proper assembly and measurement of the system, a spectrum of the He-Ne laser was found. Because the laser has a smaller wavelength range than the resolution of the system, the measurement of the laser in the spectrograph with the slit before the detector should be a sinc function, or a Fourier Transform of the size of the slit due to the diffraction from the slit. An image of this measurement is shown with the noticeable sinc function shape. 


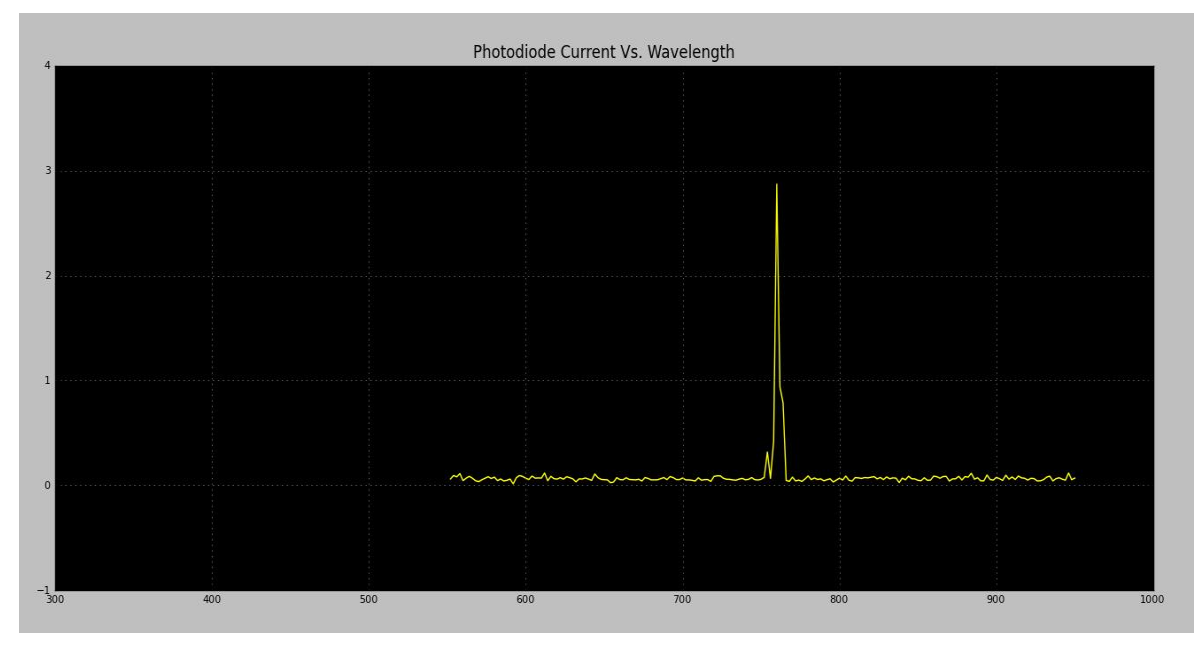

Figure 5.2: Spectrum of the He-Ne laser using the spectrograph system. Notice the sinc function, depicting the diffraction through a slit

\subsection{Final implementation}

During the competition, both experimental uses of the spectrograph were successfully tested using the competition heliostat. The data collected is shown below.

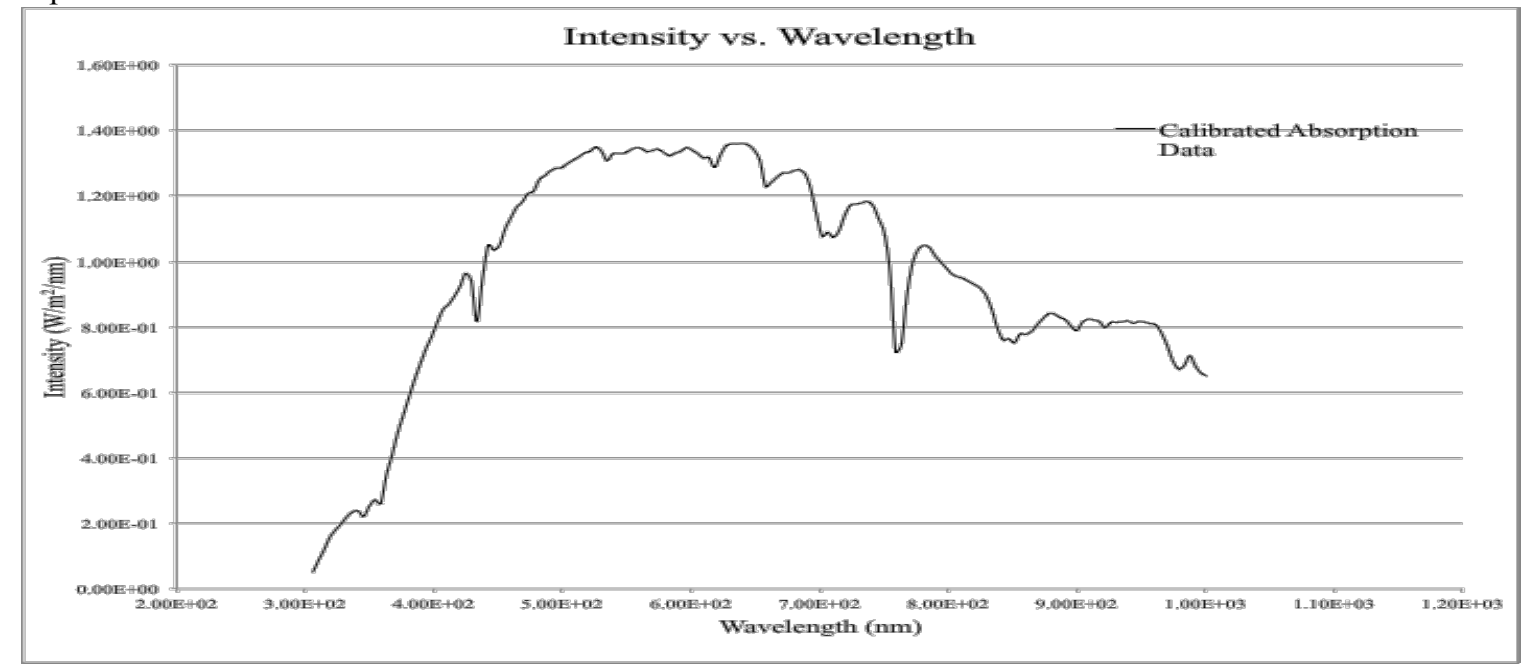

Figure 5.3: Wavelength calibration determined using water absorption at $760 \mathrm{~nm}$ and the grating zero-positioning system.

The solar spectrum data shown above implemented a wavelength calibration using the known absorption feature of water around $760 \mathrm{~nm}$ and the grating zero-positioning system as described previously. Due to inaccurate calibration data for the photodiode and grating, the data was calibrated for intensity across wavelengths using known spectral data gathered from ASTM International standards. This calibrated data is shown below compared with the known spectral data and the theoretical blackbody at $5800 \mathrm{~K}$. 


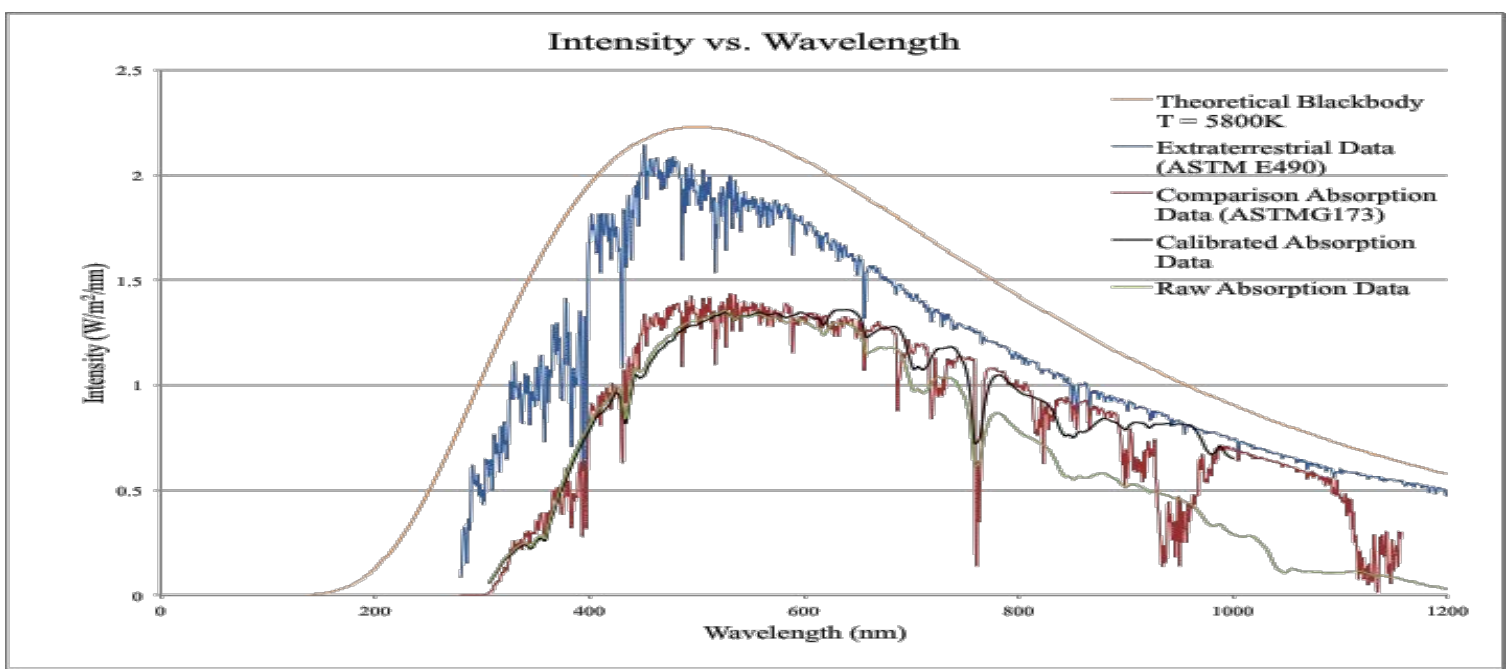

Figure 5.4: Wavelength and intensity calibrated solar spectra data compared with known solar spectra data and the theoretical blackbody at $5800 \mathrm{~K}$ and the raw spectral data.

As shown above, the calibrated solar spectra data using the spectrograph agrees closely with the known solar spectra data from ASTM International at wavelengths up to the infrared. In the infrared wavelengths, the data does not agree as closely due to the lack of sensitivity of the photodiode in these wavelengths. The calibrated data agrees more closely to the known spectral data than the uncalibrated data, demonstrating accuracy of the calibration technique.

In addition to atmospheric absorption measurements, solar imaging was also performed with the spectrograph. The graph below shows an image collected by the spectrograph the $550-565 \mathrm{~nm}$ wavelength band.

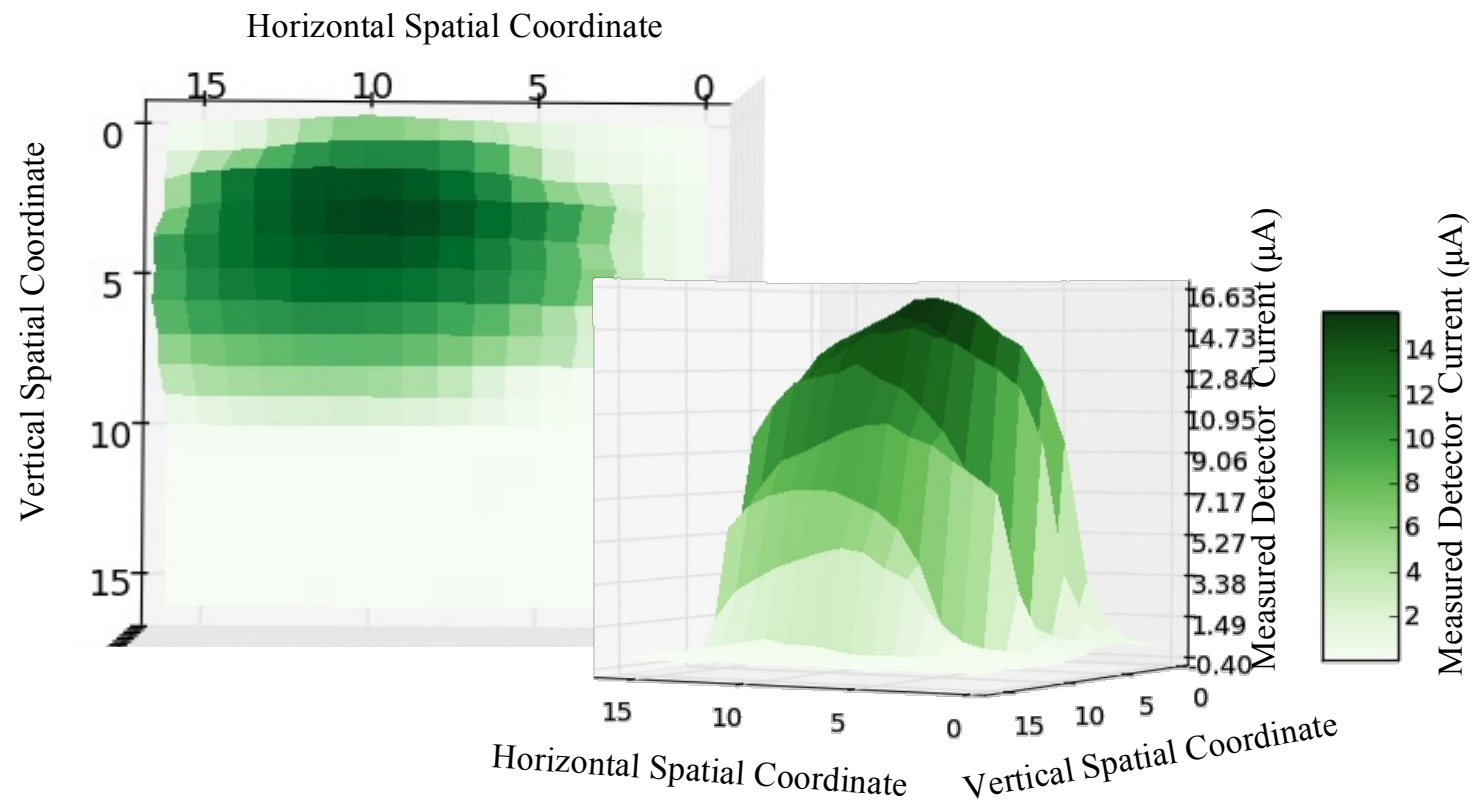

Figure 5.5: Image of the sun in the 550-565nm wavelength band. The elongation is attributed to the motion of the heliostat during the measurement period. 
The image above depicts an elongation of the sun resulting from the motion of the heliostat during the measurement period. The result of this test demonstrates the capability of imaging using a spatial filter and a photodiode rather than a CCD. Although the spatial resolution of the image above is very low, the resolution could be increased using a smaller pinhole size and changing the linear motor step size to a smaller value, demonstrating the cost effectiveness of this type of imaging over the use of a CCD. However, the data collection time using this method is significantly larger than that of a CCD, resulting in the solar elongation seen above which would not be seen using a CCD.

\section{CONCLUSION}

The project team developed and constructed a design that works for the purposes of imaging and nonimaging spectrograph applications. These two modes of operation are supported by an array of features, optics, controls, and motors. With this information in mind, the team demonstrated a spectrograph that can adequately record the spectrum of the sun, and by using a pinhole to create pixels; the spectrograph will image the sun. This capability - along with good resolution, transmission, and accuracy - gave the team an edge in the national competition, resulting in the ultimate award of Best Design of a solar spectrograph.

\section{ACKNOWLEDGEMENTS}

Through the experience granted by the National Student Solar Spectrograph Competition, each team member has developed and refined skills in the areas of optics, electronics, manufacturing, experimental testing, and data analysis applicable to future education and careers in each field. The team would like to thank Montana Space Grant Consortium for sponsoring and hosting this educational competition and for all their help throughout the year. In addition, the team would like to acknowledge all the judges who dedicated their time during the competition, as well as Richardson Gratings for their generous donation of the diffraction gratings used in the competition spectrographs.

\section{REFERENCES}

1. Schneider, Daniel, Gordon Walker, and David Gray. "Spectrographs." Reading. Web. 20 Sept. 2011. <www.tlstautenburg.de/research/artie/lectures/spectrographs2.ppt>.

2. Palmer, Christopher. Diffraction Grating Handbook. 6th ed. New York: Newport Corporation, 2005. Print.

3. James, John F. Spectrograph Design Fundamentals. Cambridge: Cambridge UP, 2007. Print.

4. Davenhall, A. C., G. J. Privett, and M. B. Taylor. "Overview of CCD Detectors." The 2-D CCD Data Reduction Cookbook. Rutherford Appleton Laboratory, 16 Aug. 2001. Web. 29 Sept. 2011. <http://starwww.rl.ac.uk/docs/sc5.htx/node7.html>.

5. Avila, G., and C. Guirao. "Slit/Pinhole Flux Calculator." Amateur Astronomical Spectroscopy. Club of Aficionados in Optical Spectroscopy, 22 May 2009. Web. 28 Sept. 2011. <http://spectroscopy.wordpress.com/2009/05/22/slitpinholeflux-calculator/>.

6. "5 - Spectroscopy." Laboratory for Atmospheric and Space Physics. Web. 05 Dec. 2011. $<$ http://lasp.colorado.edu/ bagenal/3720/CLASS5/5Spectroscopy.html $>$.

7. Arduino - ArduinoBoardMega2560." Arduino - HomePage. Arduino. Web. 06 Dec.2011. $<$ http://arduino.cc/en/Main/ArduinoBoardMega2560>.

8. "Realtime Plot of Arduino Serial Data Using Python." Blended Technologies. Blended Technologies, 8 July 2009. Web. 05 Dec. 2011.<http://www.blendedtechnologies.com/realtime-plot-of-arduino-serial-datausing-python>.

9. SfinxMagnum. Rainbow Licks WP. Digital image. Pulsar Wallpapers. Pulsar Media.Web. 9 Dec. 2011. $<$ http://www.pulsarwallpapers.com/postcard.img47222.html $>$.

10. ASTM International ASTM G173 - 03(2008) Standard Tables for Reference Solar Spectral Irradiances: Direct Normal and Hemispherical on $37^{\circ}$ Tilted Surface. $<$ http://www.astm.org/Standards/G173.htm> 